\title{
Evaluation of Circulating Protein Biomarkers of Inflammation in COPD
}

\author{
Authors \\ Shivani Jaswal ${ }^{1}$, Varinder Saini ${ }^{2}$, Jasbinder Kaur ${ }^{3}$, Seema Gupta ${ }^{4}$ \\ ${ }^{1}$ Professor, Department of Biochemistry, ${ }^{3}$ Professor, Department of Pulmonary Medicine \\ ${ }^{3}$ Professor \& Head, Department of Biochemistry, ${ }^{4}$ Assistant Professor, Department of Biochemistry \\ Government Medical College \& Hospital, Chandigarh, India \\ Corresponding Author \\ Dr Shivani Jaswal \\ Professor, Department of Biochemistry, Government Medical College \& Hospital, Chandigarh, India \\ Email:shivanirishiraj@yahoo.co.in
}

\begin{abstract}
Background: Many of the systemic manifestations of Chronic Obstructive Pulmonary Disease (COPD) are mediated through increased levels of inflammatory proteins. Hence, there is much interest in the use of biomarkers of systemic inflammation in COPD, as these may have possible application in disease phenotyping, monitoring of disease progression or exacerbation and measuring the effects of therapeutic intervention. The study was undertaken to evaluate the circulating levels of C-reactive protein (CRP) and Interleukin-8 (IL-8) in patients of COPD.

Methods: Plasma levels of CRP and IL8 were measured in 60 patients of COPD. CRP was estimated by immunoturbiditimetric method, while IL-8 levels were measured by commercially available ELISA kits. The results were expressed as mean $\pm S D$ and statistical comparison was done using student's " $t$ " test. Pearson's coefficient was used for correlation analysis.

Results: The levels of CRP and IL-8 were found to be significantly higher in patients with COPD as compared to the controls and the levels increased with the severity of the disease being significantly higher in the acute exacerbation of COPD (AECOPD) as compared to the stable COPD (CRP $=86.80 \pm 40.93,25.67 \pm 21.69 \mathrm{mg} / \mathrm{l}$ and $I L-8=97.19 \pm 55.45,21.28 \pm 15.89 \mathrm{pg} / \mathrm{ml}$, respectively). A statistically significant positive correlation ( $r=0.86, p<0.001)$ was found between the levels of CRP and IL-8 in patients of COPD, while the levels correlated negatively with FEV1\%.

Conclusion: The results of the study suggest that circulating levels of inflammatory proteins are elevated in patients of COPD and correlate positively with the disease severity. It may be concluded that levels of CRP and IL8 could be a useful complimentary criteria to improve therapeutic and prognostic strategies for COPD.
\end{abstract}

Keywords- $C O P D, C R P, I L-8$.

\section{INTRODUCTION}

Chronic obstructive pulmonary disease (COPD) is a major disease affecting the health of people globally and is expected to be the third leading cause of mortality in the world by the year $2020^{[1]}$. It is a chronic airway inflammatory disease, characterized by irreversible airflow limitation that increases progressively during the natural course of the disease.

There is increasing evidence to suggest that systemic inflammation also contributes to the morbidity associated with this disease ${ }^{[2]}$. The 
underlying molecular basis linking COPD and comorbidities is still not fully understood ${ }^{[3],[4],[5]}$. It remains unclear whether systemic inflammation is the result of systemic diffusion of local inflammation in the lungs, or is a consequence of comorbid conditions, which impact on lungs. One possible mechanism may be due to the spill-over of pro-inflammatory factors and cells from lung to peripheral blood, probably due to the increased permeability of pulmonary vessels in $\mathrm{COPD}^{[6]}$. Another hypothesis supports the proposition that an enhanced expression of granulocyte-macrophage colony-stimulating factor and IL-6 in COPD plays an important role, as these mediators stimulate neutrophils release from the bone marrow causing an increased neutrophil amount in the peripheral blood $^{[7],[8]}$. Systemic hypoxia due to progression of COPD has been suggested as a possibility ${ }^{[2]}$.

Most of the systemic manifestations are likely the result of inflammatory processes and several biomarkers such as various cytokines, adipokines, C-reactive protein and coagulation factors have been investigated for their role in inflammation ${ }^{[9-12]}$. The standard method for classifying disease severity is the measurement of forced expiratory volume in 1 second (FEV1) ${ }^{[13]}$. However, there is a need for biomarkers that are reflective of the inflammatory mechanisms involved in disease pathogenesis. Such biomarkers may be useful for monitoring disease progression, evaluating the effects of therapeutic interventions or identifying disease sub-phenotypes with different clinical characteristics. The study was thus, planned to evaluate the biomarkers of systemic inflammation in patients with COPD.

\section{MATERIAL AND METHODS}

The study group consisted of 60 patients of COPD, meeting the diagnostic criteria of the GOLD for COPD $^{[14]}$. The subjects were divided in two groups based on the disease severity. 30 patients had acute exacerbation (AECOPD) while 30 patients had stable COPD ie those who had not been on any glucocorticoid or antibiotic treatment for the last four weeks prior to recruitment. Patients with concomitant pneumonia or any other respiratory disease were excluded from the study. 30 healthy age and sex matched volunteers were recruited from the hospital staff to serve as control group. Healthy controls had no history of smoking, chronic underlying illness, no recent upper airway infection and no respiratory symptoms like cough, wheezing etc. Clearance from the Institutional Research and Ethics Committee was obtained and informed consent was taken from all the participants of the study.

Blood samples were drawn from all participants at the time of recruitment for estimation of C-reactive protein and IL-8. C-reactive protein was estimated by immunoturbiditimetric method on Modular $\mathrm{P}$ 800 random access chemistry analyzer, while IL-8 levels were measured by commercially available ELISA kits, according to manufacturer's instructions. Pulmonary function tests were performed on all patients and controls using standardized methods on SPIROLAB III.

The results were expressed as mean \pm SD and statistical comparison was done using student's " $t$ " test. Pearson's coefficient was used for correlation analysis. The difference was considered significant when $\mathrm{p}<0.05$.

\section{RESULTS}

There was no statistically significant difference in the age and duration of the the disease between patients of AECOPD and those with stable COPD. The levels of C-reactive protein and IL-8 were found to be significantly higher in patients with COPD as compared to the controls and the levels increased with the severity of the disease being significantly higher in the AECOPD as compared to the stable COPD. (Fig.1)

A statistically significant positive correlation $(\mathrm{r}=0.86, \mathrm{p}<0.001)$ was found between the levels of C-reactive protein and IL- 8 in patients of COPD, while the levels correlated negatively with FEV1\% (Fig 2 \& Fig. 3 respectively). 
Fig1. Levels of CRP \& IL-8 in patients of COPD.
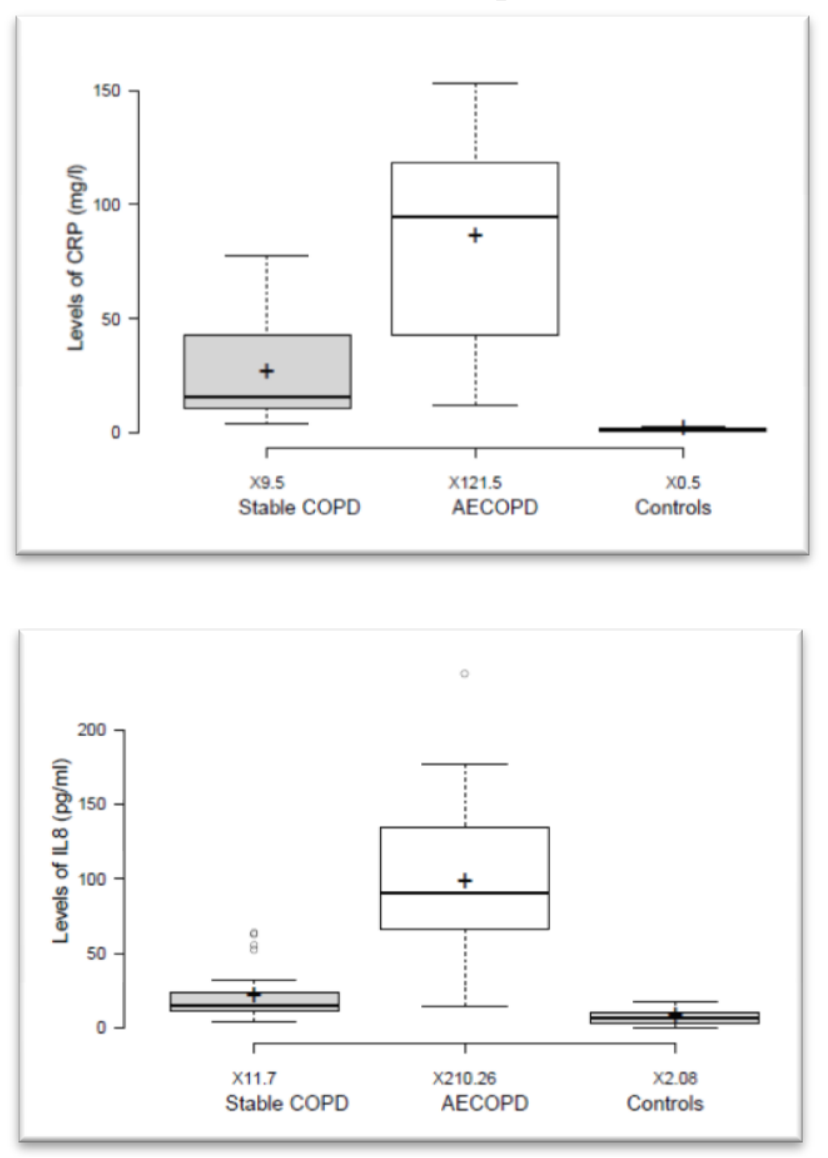

Fig 2: Correlation between levels of CRP and IL-8 in patients of COPD.

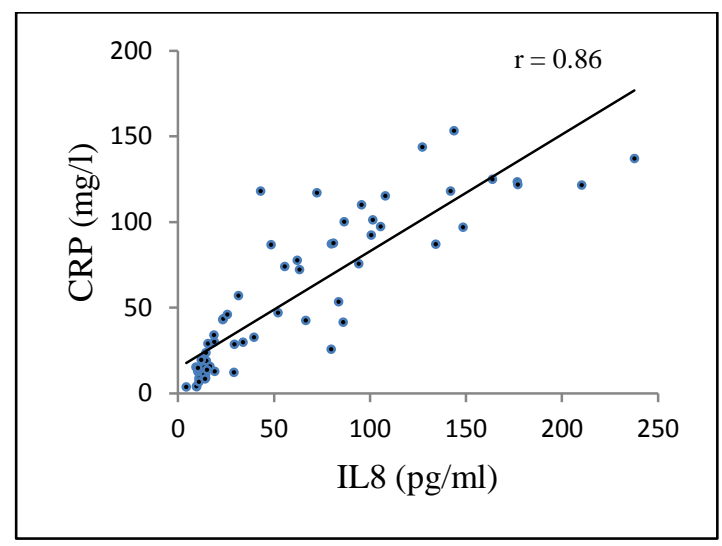

Fig 3: Correlation between levels of CRP \& IL-8 with lung function in patients of COPD.

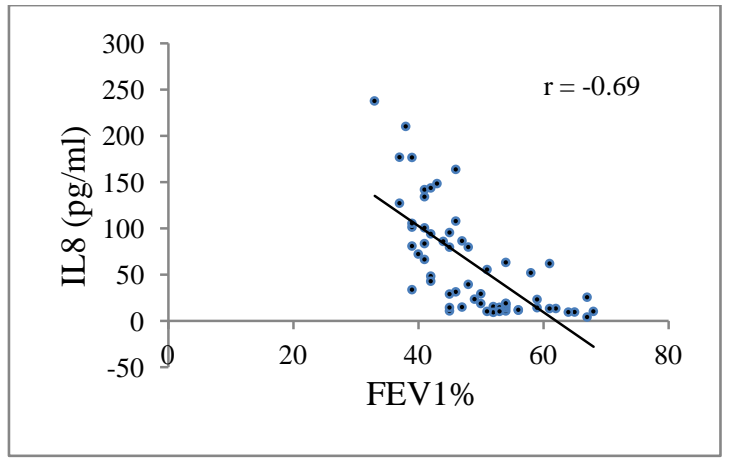

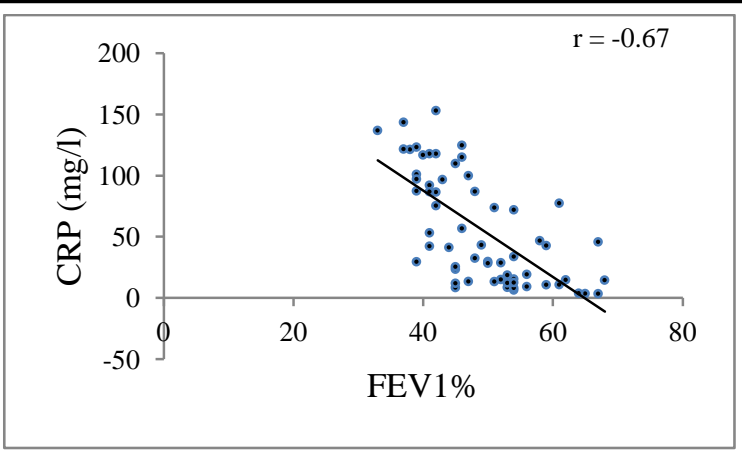

\section{DISCUSSION}

COPD is characterized by an intense inflammatory process in the airways, parenchyma, and pulmonary vasculature. The systemic manifestations of COPD are recognized, but the understanding of their etiology and importance is lacking. Evidence indicates that airway inflammation is mediated through a variety of cells, including macrophages, neutrophils and T \& B lymphocytes by the secretion of a number of pro-inflammatory cytokines ${ }^{[15-17]}$. One of the major cell types involved in inflammatory events associated with COPD is alveolar macrophages, which are able to produce several chemotactic factors ${ }^{[18,19]}$. The high production of chemo-attractants results in increased lung neutrophil and lymphocyte infiltration which in turn produce pro-inflammatory cytokines that contribute to the development and progression of the lung disease. The chronicity of COPD systemic inflammation is sustained by an increased production of several pro-inflammatory cytokines at both serum and airway levels.

The main cytokines involved in the systemic inflammation of COPD are IL-8, IL- 6 and TNF- $\alpha$. Among them, IL- 8 is the most important, which can activate neutrophils and cause degranulation ${ }^{[20,21]}$. Various inflammatory mediators are released in the degranulation, promoting inflammatory reaction. IL-8 may be an important inducible factor of CRP and other protein produced in the acute phase of inflammation, and hence, involved in the systemic inflammation of COPD.

In the present study the levels of markers of systemic inflammation, ie, CRP and IL-8 were found to be significantly higher in patients of COPD as compared to controls. Moreover, patients with AECOPD had the highest plasma IL-8 and CRP 
levels as compared to patients with stable COPD and healthy controls, in line with certain previous publications $^{[22,23]}$. Previously it has been demonstrated that IL-8 levels are increased in the alveolar lavage fluid in COPD patients, and that its increased levels in sputum samples correlated with the airway bacterial load and proteinase released from activated neutrophils ${ }^{[24]}$. IL-8 levels in induced sputum have also been found to be correlated with the extent of neutrophilic inflammation and with disease severity (\%predicted FEV1) ${ }^{[25]}$.

Increased levels of IL8 in the serum, suggest that it may act is a selective attractant of neutrophils, and may play a role in the initiation of an acute phase response through the acute phase proteins including CRP.

A significant positive correlation was found between levels of IL-8 and CRP which indicates that that these inflammatory mediators may be interregulated. The positive correlation may suggest that IL-8 may possibly promote CRP production in COPD patients.

Levels of IL-8 and CRP were found to be negatively correlated with FEV1\% suggesting that systemic inflammation might play a role in the development of airway obstruction, morbidity and severity.

\section{CONCLUSION}

In conclusion, this study demonstrated that both serum IL-8 and CRP levels were significantly higher in patients of COPD and their levels correlated positively with the severity of the disease. The results suggest that these levels could be useful complimentary criteria to improve therapeutic and prognostic strategies for COPD.

As the study was limited by sample size, further studies with larger sample size may be required to validate this result

\section{ACKNOWLEDGEMENT}

The financial grant provided by the Department of Science and Technology, Chandigarh Administration for carrying out the study is deeply acknowledged.

\section{REFERENCES}

1. Murray CJ, Lopez AD. Alternative projections of mortality and disability by cause 1990-2020: Global burden of Disease Study. Lancet 1997; 349:1498-1504.

2. Chan KH, Yeung SC, Yao TJ, Ip MSM, Cheung AHK, Yeung MMW, Mak JCW. Elevated plasma adiponectin levels in patients with COPD. Int J Tuberc Lung Dis 2010;14(9): 1193-1200

3. Nussbaumer OY, Rabe KF.Systemic manifestations of COPD. Chest 2011;139: 165-173.

4. Corbi G.M, Carbone S, Ziccardi P, Giugliano G, Marfella R, Nappo, F, etal. FFAs and QT intervals in obese women with visceral adiposity: Effects of sustained weight loss over 1 year. J. Clin. Endocrinol. Metab. 2002;87:2080-2083.

5. Corbi GM, Bianco A, Turchiarelli V, Cellurale M, Fatica F, Daniele A etal. Potential mechanisms linking atherosclerosis and increased cardiovascular risk in COPD: Focus on sirtuins. Int I Mol Sci 2013;14:12696-12713.

6. Barnes PJ, Celli BR. Systemic manifestations and comorbidities of COPD. Eur Respir J 2009;33:1165-1185.

7. Laveneziana P, Palange P. ERS research seminar faculty. Physical activity, nutritional status and systemic inflammation in COPD. Eur Respir J 2012;40:522-529.

8. Vaguliene N, Zemaitis M, lavinskiene S, Miliauskas S, sakalauskas $\mathrm{R}$. Local and systemic netrophilic inflammation in patients with lung cancer and COPD. BMC Immunol 2013;14:36.

9. Wouters EF, Groenewegen KH, Dentener MA, Vernooy JH. Systemic inflammation in chronic obstructive pulmonary disease:the role of exacerbations. Proc Am Thorac Soc.2007;4(8):626-34.

10. Broekhuizen R, Wouters EF, Creutzberg EC, Schols AM. Raised CRP levels mark metabolic and functional impairment in advanced COPD. Thorax 2006;61(1):17-22. 
11. Yende S, Waterer GW, Tolley EA, et al. Inflammatory markers are associated with ventilator limitation and muscle dysfunction in obstructive lung disease in well functioning elderly subjects. Thorax 2006;61:10-16.

12. Higashimoto $\mathrm{Y}$, Yamagata $\mathrm{Y}$, Taya $\mathrm{S}$. Systemic inflammation in chronic obstructive pulmonary disease and asthma: Similarities and differences. Respirology 2008;13(1):128:133.

13. Xie J, Yang X-Yi, Shi J, Deng X, Long W. A new inflammation marker of chronic obstructive pulmonary disease-adiponectin. World J Emerg med 2010;1(30):190-195.

14. Global strategy for the diagnosis, management and prevention of COPD. Global initiative for chronic obstructive lung disease

(GOLD)2010;http://www.goldcopd.org

15. Whiteman SC, Bianco A, Knight RA, Spiteri MA. Human rhinovirus selectively modulates membranous and soluble forms of its intracellular adhesion molecule (ICAM-1) receptor to promote epithelial cell infectivity. J Biol Chem 2003;278:11954-11961.

16. De Laurentiis G, Paris D, Melck D, Montuschi P, Maniscalco M, Bianco A etal. Separating smoking related diseases using NMR based metabolomics of exhaled breath condensate. J Proteome Res 2013;12:15021511.

17. Bianco A, Whiteman SC, sethi SK, Allen JT, Knight RA, Spiteri MA. Expression of intercellular adhesion molecule-1 in nasal epithelial cells of atopic subjects: a mechanism for increased rhinovirus infection?. Clin Exp Immunol 2000;121:339-345.

18. Corsonello A, Antonelli Incalzi R, Pistelli R, Pedone C, Bustacchini S, Lattanzio F. Comorbidities of chronic obstructive pulmonary diseaseCurr Opin Pulm med 2011;17:S21-S28.

19. Agusti A. Systemic effects of COPD: what we know and what we don't know (but should). Proc Am Thorac Soc 2007;4:522525.

20. Bhowmick A, Seemungal TA, sapsford RJ, Wedzicha JA. Relation of sputum inflammatory markers to symptoms and lung function changes in COPD Exacerbations. Thorax 2000;55:114-120.

21. Ehling A, Scgaffer A, Herfarth H, Tarner IH, Anders S, Distler $\mathrm{O}$ etal. The potential of Adiponectin in driving arthritis. I Immunol 2006;176:4468-4478.

22. Man SF, Connet JE, Anthonisen NR, Wise RA, Tashkin DP, Sin DD. C-reactive protein and mortality in mild to moderate COPD. Thorax 2006;61:849-853.

23. Pinto-Plata VM, Mullerova $\mathrm{H}$, Toso JF etal. C-reactive protein in patients with COPD, control smokers and non-smokers. Thorax 2006;61:23-28.

24. Serum and bronchial lavage fluid concentrations of IL-8, SLPI, sCD14 and sICAM-1 in patients with COPD and asthma. Camilla Hollandera, Brigita Sitkauskieneb, Raimundas Sakalauskasb, Ulla Westina, Sabina M. Janciauskiene. Respiratory Medicine (2007) 101, 1947-1953

25. Yamamoto C, Yoneda T, Yoshikawa M, Fu A, Tokuyama $\mathrm{T}$, Tsukaguchi $\mathrm{K}$, et al. Airway inflammation in COPD assessed by sputum levels of interleukin-8. Chest 1997;112(2):505-10. 